\title{
Statistical Characterisation of Metals from Ultrasonic Aberrations
}

\author{
J. A. Hernández*, M. Clark, I. J. Collison, M. Somekh, S. D. Sharples \\ School of Electrical \& Electronic Engineering \\ University of Nottingham \\ University Park, \\ Nottingham, UK \\ NG7 2RD. \\ *e-mail : eexjah@nottingham.ac.uk
}

\begin{abstract}
Materials with random microstructure can have an adverse effect on ultrasonic measurements through scattering and aberration of the acoustic field. We have developed a theoretical and experimental technique for characterising the effect of the material microstructure on the propagation of acoustic waves. The theoretical method predicts propagation of the statistical properties in the random medium. Using this model and a novel experimental technique we can extract materials properties from observations of acoustic aberrations. The microstructure has been modelled as having grains randomly orientated and weakly anisotropic. Moreover, individuals grains are treated to be equiaxed. This is summarised by assuming that the correlation function for the wave number has a Gaussian shape. Under this assumption, the approximated power correlation function for the acoustic field has been obtained using the stochastic wave equation for random media, along with numerical simulation using phase screen theory. The experimental evidence that aberrations are frequency dependent is presented. Multiple cscans on titanium were performed using a $10 \mathrm{MHz}$ transducer as an ultrasonic source. The output of the transducer gives substantial frequency components between $6 \mathrm{MHz}$ and $16 \mathrm{MHz}$. The variation of the frequency on a fixed sample enables us to examine different measurement regimes (higher frequencies correspond to large grains). The statistical analysis and estimated power correlation function from measurements are compared to a modelled power correlation function. The correlation length and standard deviation of the wave number define the power correlation function of the field. This function has been fitted to the estimated power correlation from measurements on titanium 6-4, and values for the variance and correlation length were obtained. The paper demonstrate that the stochastic model is capable of quantitative prediction of the predominant wave scattering effect in granular materials.
\end{abstract}

\section{INTRODUCTION}

Acoustic aberrations are important in surface wave measurements. It has been realised that aberrations affects the accuracy of measurements [1]. Over the years an optical scanning acoustical microscope (O-SAM) has been developed for detecting and acoustic adapting of aberrations in polycrystalline materials, [1], [2]. The aim of this paper is to show the experimental evidence of acoustic aberration and to study them from a statistical point of view. That is, since most engineering materials like titanium have random microstructure (crystal or grains randomly orientated) the response to propagation of acoustic waves will be random. The grain microstructure may have various effects on ultrasonic wave propagation. It attenuates, scatter and aberrates ultrasonic waves as they propagate within this medium. Generally speaking aberrations are wavefront distortion of ultrasonic waves propagating in random medium. It would be desirable to quantify the strength of aberrations on a material with random microstructure. The main observation relies on the transverse coherence of the acoustic field as it propagates. The first step is to measure aberrations then the strength by means of transverse power correlation function of the acoustic field, which will be the subject of forthcoming sections. The theoretical description of SAW propagation is based on a scalar stochastic theory for waves in a random medium. Therefore it is possible to obtain an expression for second order moments of the field which carries information of microstructure. The behaviour of power correlation is mainly dictated by the scale of the inhomogeneity which is a rough estimation of grain size. The theoretical derivation is not included in this paper as the main purposes is to show how this technique behaves under different regimes of frequency and also because it has been developed in a previous paper, [3].

\section{ABERRATIONS IN METALS}

Fig. 1 shows several speckle patterns obtained with experimental arrangement shown in Fig. 3. It is well known that microstructure or grain size is sensitive to frequency. The frequency output of the transducer where aberrations can be observed lies between $5 \mathrm{MHz}$ and $16 \mathrm{MHz}$ approximately. The idea is to test the theoretical power correlation within this range.

The first column shows the amplitude distribution of the acoustic field whereas second column shows the residual phase. The important point here is to observe how the amplitude breaks up with distance as well as phase variations, where propagation is from left to right. The fundamental frequency is $10 \mathrm{MHz}$ so analysis of the speckle pattern at that frequency is expected to provide the most accurate estimation of mean grain size. 

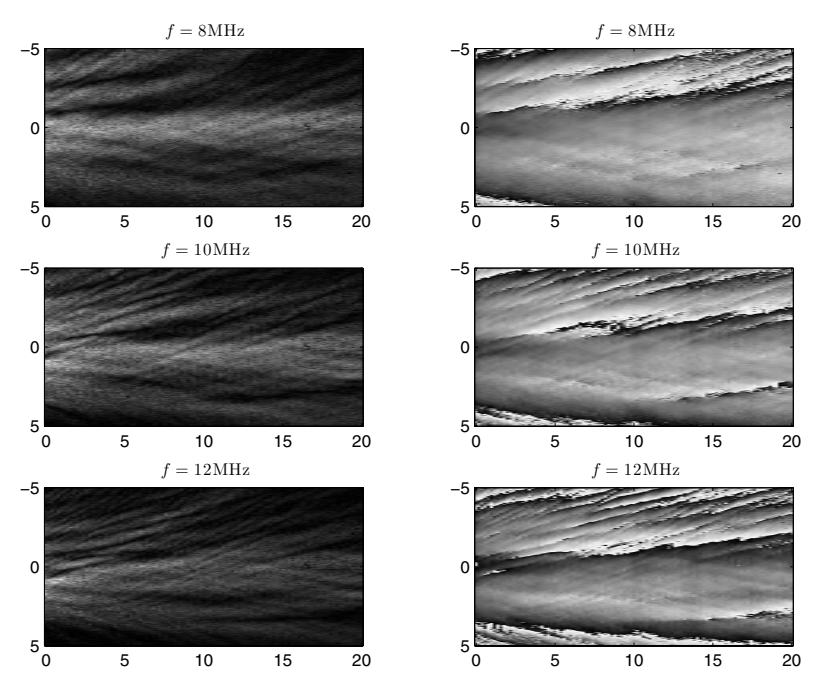

Fig. 1. Amplitude and phase distribution of the acoustic field $u_{y}(\boldsymbol{r}, \omega \mid \gamma)$ showing aberrations in $\mathrm{Ti}$ at different frequencies. The propagation is from left to right.

As it can be observed the speckle patterns are slightly different as the acoustic field interacts at different frequencies with the grains. The differences are more clearly observed with the estimated power correlation at each frequency. This statistical analysis is been done in section III as it requires some mathematical definitions.
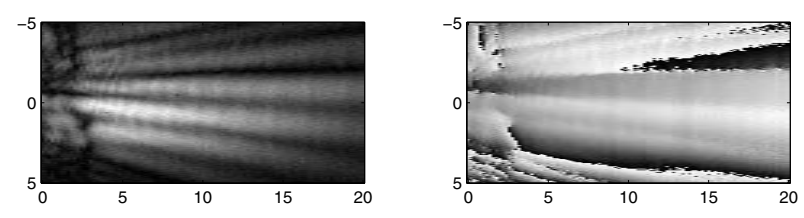

Fig. 2. Amplitude and phase distribution of the acoustic field at $10 \mathrm{MHz}$ on an ideal sample (Glass) with no microstructure. The transducer output is not a single beam as one might expect.

The aberrations in these set of experiments turned out to be weak as the output beam dominates propagation. Comparison can be made with Fig. 2 where is it shown phase and amplitude distribution on an ideal sample.

\section{A. Experimental setup}

In this experiment a $10 \mathrm{MHz}$ transducer has been used as the source to generate SAW within the material Fig. 3(a). Multiple c-scan measurements were performed over the surface of a piece of titanium-alloy. The source was relocated at multiple positions on surface and a c-scan was performed each time Fig. 3b,c. The purpose was to make the ultrasonic field interact with different grain sizes and to build an ensemble of the acoustic field. Therefore, for each point $\boldsymbol{r}=(x, z)$ and fixed source position a time-waveform, $u_{y}(\boldsymbol{r}, t)$ for the vertical displacement was obtained. The displacement $u_{y}(\boldsymbol{r}, t)$ has been detected using a modified knife-edge detector, [2]. Then the data were acquired using a oscilloscope Fig. 3(b). (a)
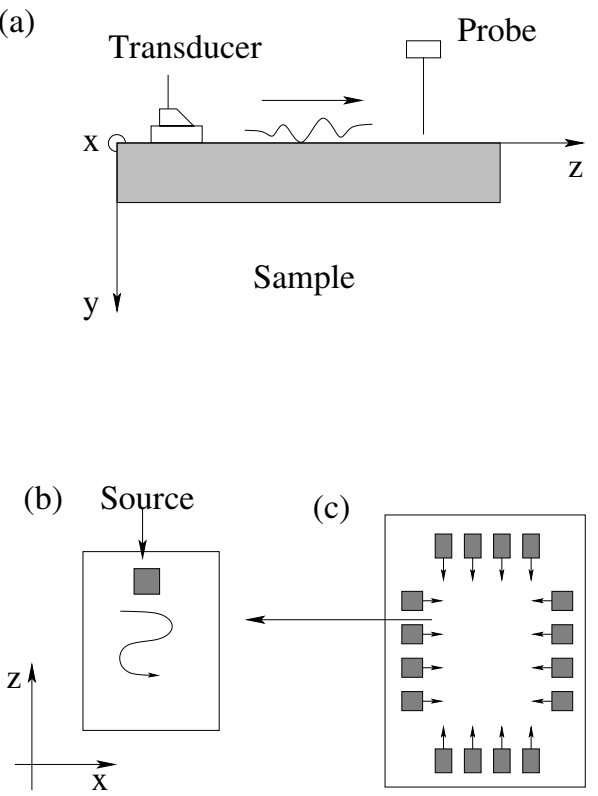

Fig. 3. (a) Sample and transducer array for SAW generation. (b) Area in $x z$ plane which c-scan has been performed. (c) The black square represents the multiple positions where transducer has been located to perform a c-scan according diagram (b); whereas arrows indicates direction of propagation.

The waveform $u(\boldsymbol{r}, t \mid \gamma)$ has been transformed to the frequency domain using Fourier transform,

$$
u_{y}(\boldsymbol{r}, \omega \mid \gamma)=\int u_{y}(\boldsymbol{r}, t \mid \gamma) e^{-i \omega t} d t
$$

where $\gamma$ represents a sample of the acoustic ensemble across microstructural ensemble.

\section{Data Statistical AnAlysis}

The discussion is based on statistical concepts such as the second order moment or energy correlation function for finite sequences. Notation will be introduced to explain some of the key concepts so that a comparison between the theory earlier developed in paper, [3] and experiments can be achieved. The $x z$ plane has been divided into a grid of size $m \times l$ for data acquisition. The pixel size is $25 \mu \mathrm{m}$ in $x$-direction and $1000 \mu \mathrm{m}$ in $z$-direction. The displacement $u_{y}$ at every grid point is being denoted by $U_{k l}^{\gamma}$ and analysis applies for each propagation distance. By definition the cross-correlation is $\left\langle U_{k l}^{\gamma} U_{k^{\prime} l}^{\gamma *}\right\rangle$ where \langle\rangle denotes the ensemble average. The estimation of the ensemble average of $Z_{k k^{\prime} l}^{\gamma}=U_{k l}^{\gamma} U_{k^{\prime} l}^{\gamma *}$ is rather complicated since there is little statistical information about $U_{k l}$. Instead, two different averages will be performed. By making $m=k-k^{\prime}, Z_{k k^{\prime}}$ it can be rewritten as $Z_{k(k+m) l}^{\gamma}=U_{k l}^{\gamma} U_{(k+m) l}^{\gamma *}$. Since there is a random function for each $m$, the average over $k$ is performed as well as the ensemble average, leading to

$$
\begin{aligned}
\left\langle Z_{m l}^{\gamma}\right\rangle & =\left\langle\frac{1}{|\gamma|} \sum_{k}^{|\gamma|} Z_{k(k+m) l}^{\gamma}\right\rangle \\
& =\frac{1}{|\gamma|} \sum_{k}^{|\gamma|}\left\langle Z_{k(k+m) l}^{\gamma}\right\rangle
\end{aligned}
$$

Here $|\gamma|$ is the total number of measurements. $\left\langle Z_{m}^{\gamma}\right\rangle$ remains a complex function so its modulus will be considered, and it 
will be termed as the power correlation function; hence:

$$
\Gamma_{m l}^{e}=\left|\left\langle Z_{m l}^{\gamma}\right\rangle\right|
$$

The measured correlation function between finite signals, $\Gamma_{m l}$ implemented numerically is shown in Fig. 4. The importance of this function will become apparent when it is compared to the theoretical. The overall decay of this function is strongly related to aberrations due to the interaction between the ultrasound and grain structure.
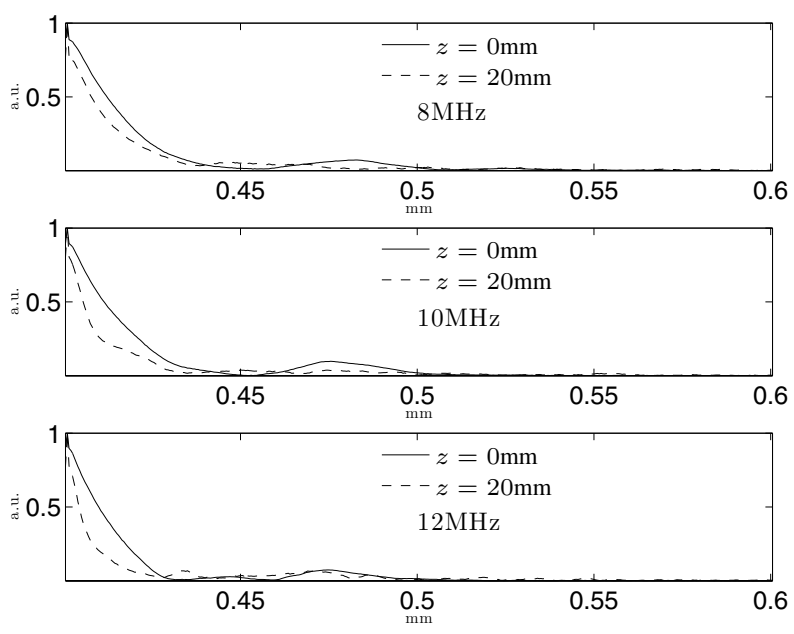

Fig. 4. Power correlation function at different frequencies showing how weakly it decay with didstance

\section{A. Theoretical power correlation}

Expression (3) is to be compared with a theoretical power correlation of $u_{y}$ developed in [3]. The mathematical definition of transverse correlation for $u_{y}$ is as follows,

$$
\Gamma_{u_{y}}=\int\left\langle u_{y}(x, z, \omega \mid \gamma) u_{y}^{*}(x+\tau, z, \omega \mid \gamma)\right\rangle d x
$$

where $\tau=x-x^{\prime}$. The propagation of integrand in (4) in a random medium has been the subject of several papers in subjects other than ultrasonics, [4]-[8]. Nevertheless, an approximate solution to propagation of the second order moment through random media has been obtained in [3]. The approximation is based on a phase screen theory, [9] and the stochastic wave equation. The main assumption is that the approximated wave velocity variation can be accounted for by Gaussian random function whereas aberrations are quantified by means of standard deviation. That it is to say, an acoustic wave propagates in a random medium according to the stochastic wave equation and wave number $k(\boldsymbol{r}, \gamma)=k_{R}(1+\mu(\boldsymbol{r}, \gamma))$. Therefore variations of velocity with respect to $k_{R}$ are accounted for as $\sigma^{2}=\left\langle\frac{\left(k-k_{R}\right)^{2}}{k_{R}^{2}}\right\rangle$ where $\sigma=\sqrt{\left\langle\mu^{2}\right\rangle}$ is the root mean square. $k_{R}$ represents the background wave number corresponding to the surface wave velocity in homogeneous medium. In order to obtain an exact expression for $\Gamma_{u_{y}}$ the microstructure has been assumed to have equiaxed grains. This is equivalent to assume that the second moment for $\mu$ takes the form

$$
\left\langle\mu(\boldsymbol{r}, \gamma) \mu\left(\boldsymbol{r}^{\prime}, \gamma\right\rangle=\sigma^{2} e^{-\frac{\left|r-r^{\prime}\right|^{2}}{l_{x}}}\right.
$$

where $l_{x}$ is the correlation length of $\mu$, i.e. $l_{x}$ is the minimum distance where two points $\boldsymbol{r}, \boldsymbol{r}^{\prime}$ are no longer correlated. In fact, $l_{x}$ is the distance where the function in (5) drops to $\frac{\sigma^{2}}{e}$. $2 l_{x}$ is approximately a measure of the mean grain size of the material.

Under the above conditions an exact expression for $\Gamma_{u_{y}}$ is obtained and bears the following form

$$
\Gamma_{u_{y}}=\Gamma_{0} \exp \left[-p_{n}\left(\sigma^{2}, l_{x}\right)\left(1-e^{-\frac{\tau^{2}}{l_{x}^{2}}}\right)\right]
$$

where $p_{n}=\frac{\sqrt{\pi} k_{R}^{2}}{4} l_{x} \sigma^{2}(n \Delta z)$ and $\Gamma_{0}$ is the power correlation of the field at the source location. The function $p_{n}$ depends on various parameters but from the above discussion the important parameters here are $l_{x}$ and $\sigma^{2}$ as they dictate the behaviour of $\Gamma_{u_{y}}$. The term $n \Delta z, n$ an arbitrary integer, in fact represents the propagation distance at which $\Gamma_{u_{y}}$ is to be propagated in this approximation; thus $z=n \Delta_{z}$. The other terms are constant except for the wave number $k_{R}$ which obviously depends on frequency.

\section{RESULTS}

There are two parameters which are free in (6), being $\sigma^{2}$ and $l_{x}$. These have been obtained by fitting $\Gamma_{m l}^{e}$ to $\Gamma_{u_{y}}$ by using a non-linear least square algorithm. The results for some representatives frequencies are presented in table I

TABLE I

PARAMETERS FOR STRENGTH OF ABERRATIONS

\begin{tabular}{|c||c|c|c|c|c|c|}
\hline$\omega(\mathrm{MHz})$ & 8 & 9 & 10 & 11 & 12 & 13 \\
\hline$\sigma$ & 0.024 & 0.025 & 0.028 & 0.028 & 0.026 & 0.027 \\
\hline $2 l_{x}(\mu \mathrm{m})$ & 351 & 399 & 368 & 428 & 424 & 424 \\
\hline
\end{tabular}

Fig. 5 shows a comparison of the predicted and measured power correlation function with values according to table I. The fuzzy behaviour at the end of lines in Fig. 5 is due to noise. Their disagreement in that region is unimportant since the graphs fit quite well otherwise. The agreement out of the fuzzy region means that the width and decay ratio of (3) is well predicted using the stochastic model described by (6).

The weak aberration is reflected in the power correlation where it decays slowly with distance Fig. 4. It should be expected, at least theoretically in highly aberrated materials, that the power correlation decays and gets narrow as it propagates. It can be said that the acoustic field is interacting with grains. Otherwise, the propagation will imitate propagation in homogeneous materials.

The parameter $2 l_{x}$ characterise material microstructure but the metallographic study of the sample tested did not clearly reveal the grain boundaries so the values in table I could not be satisfactorily validated using standards techniques. It is worth mentioning that in paper, [3] where aluminium was tested, the parameters are in satisfactory agreement with the actual measured microstructure. 

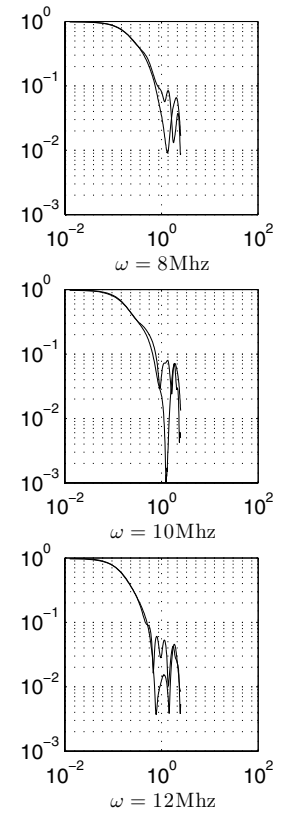
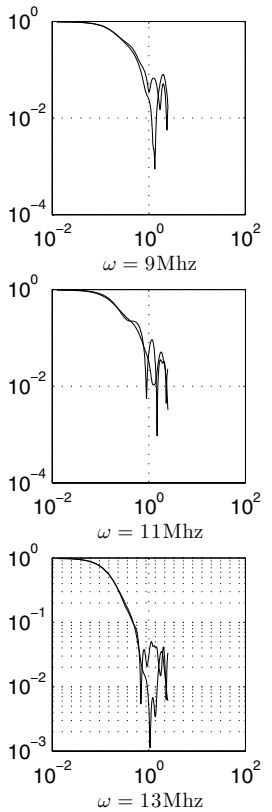

Fig. 5. LogLog Comparison of measured power correlation (3) and (6)

\section{CONCLUSIONS}

A statistical technique has been presented that can serve as a basis for material characterisation with random microstructure. The analysis has been based on a stochastic approach as the medium has been treated as if it were a random medium. We have obtained parameters that characterise the anisotropy and length scale of the materials at different frequencies. The multiple frequency interaction gives information about different length scales present in the material. In future work similar measurements will be carried out throughly characterised material grain structure.

\section{ACKNOWLEDGEMENT}

I would like to thank The University of Nottingham for the funding provided, The Engineering and Physical Science Research Council (EPSRC), the UK centre for NDE and to Rolls Royce for the samples provided to carry out this work.

\section{REFERENCES}

[1] M. Clark, S. D. Sharples, and M. G. Somekh, "Optimisation using measured green's function for improving spatial coherence in acoustic measurements," Ultrasonics, vol. 42, pp. 205-212, 2004.

[2] S. D. Sharples, M. Clark., and M. G. Somekh, "All-optical adaptive scanning acoustic microscope," Ultrasonics, vol. 41, pp. 295-299, 2003.

[3] J. A. Hernández, M. C. ans S. Sharples, M. Somekh, and V. Lopez, "Aberrations in polycrystalline materials," Submitted to J. Acoust. Soc. Am. (On review), 2005.

[4] D. A. de Wolf, "Backscatter corrections to the parabolic wave equations," J. Opt. Soc. Am., vol. 6, no. 2, pp. 174-179, 1989.

[5] K. Sobczyk, Stochastic wave propagation. Krakowskie Przedmiescie 7,00-068, Warszawa, Poland: PWN-Polish Scientific Publishers, 1984.

[6] A. Ishimaru, Wave Propagation and Scattering in Random Media. 111 Fifth Avenue, New York 10003: Academic Press,Inc, 1970.

[7] C. L. Rino, "On propagation in continuous random media," Waves in random media, vol. 2, pp. 59-72, 1991.

[8] L. Mandel and E. Wolf, Optical coherence and quantum optics. Cambridge University Press, 1995.

[9] J. W. Goodman, Statistical optics. JOHN WILEY AND SONS, INC., 2000. 